\title{
Cooling of a rotating strange star in the color superconducting phase with a crust
}

\author{
Y. W. Yu and X. P. Zheng
}

Institute of Astrophysics, Huazhong Normal University, Wuhan 430079, PR China

e-mail: zhxp@phy.ccnu.edu.cn

Received 9 September 2005 / Accepted 14 December 2005

\section{ABSTRACT}

Aims. We investigate the thermal evolution of strange stars in the 2-flavor color superconductivity and color-flavor locked phases under the influence of deconfinement heating.

Methods. Due to the spin-down of strange stars, the nuclear matter at the base of the thin crusts dissolves into quarks, releasing energy to heating the stars. On the other hand, the neutrino emissivities and specific heat involving pairing quarks are suppressed by the large pairing gap in color superconducting phases. Then the thermal evolution equation of strange stars is calculated.

Results. Deconfinement heating delays the cooling of strange stars considerably. The presence of color superconductivity with a large gap enhances this effect. Especially, in the color-flavor locked phase, the stars cannot be very cold at an early age but they cool slowly. For the stars with strong magnetic fields, a significant heating period could exist during the first several ten or hundred years. In addition, we reckoned that a possible theoretical limit line, which is determined by the competition between deconfinement heating and surface photon cooling, may indicate the upper limit temperature that isolated compact stars should not exceed.

Conclusions. Deconfinement heating is important for the thermal evolution of strange stars and is especially determinant for the stars in color-flavor locked phase which could show characteristic cooling behavior under this heating effect.

Key words. stars: neutron - stars: evolution - dense matter - pulsars: general

\section{Introduction}

Cooling simulation based on interior physics is of significant interest for the research of compact stars. In accordance with nuclear physics, a quark matter core could be produced in the interior of compact stars (hybrid star) and even strange quark matter stars (strange star, SS) may exist. Phenomenological and microscopic studies have confirmed that quark matter at a sufficiently high density, as in compact stars, undergoes a phase transition into a color superconducting state, which are typical cases of the 2-flavor color superconductivity (2SC) and colorflavor locked (CFL) phases (Shovkovy 2004; Alford 2004). Theoretical approaches also concur that the superconducting order parameter, which determines the gap $\Delta$ in the quark spectrum, lies between 10 and $100 \mathrm{MeV}$ for baryon densities existing in the interiors of compact stars. Recently, the cooling of hybrid stars with color superconducting quark cores have been investigated. Stars with CFL cores behave similarly to ordinary neutron stars (Shovkovy \& Ellis 2002). And with a designed $2 \mathrm{SC}+\mathrm{X}$ phase, hybrid stars could also explain the cooling data properly (Grigorian et al. 2005). In these cases, the thermal properties of the quark cores are suppressed by the large gap, and the hadronic matter parts of the stars play an important role in their cooling history.

The thermal evolution of SSs has also been extensively discussed. In early works, it was generally accepted that the surface temperature of SSs should be lower than neutron stars at the same age due to the quark direct Urca (QDU) processes (Alcock et al. 1988; Pizzochero 1991; Page 1992; Schaab et al. 1996). However, since the electron fraction could be small or even vanish, the QDU processes may be switched off. The cooling of SSs dominated by the quark modified Urca (QMU) and quark bremsstrahlung (QB) processes can be slower than neutron stars with standard cooling (Schaab et al. 1997a,b). Of course, a color superconducting phase could occur in SSs, and its effect on the cooling of the stars is a significant issue. Blaschke et al. (2000) show that the cooling of the stars in the 2SC phase (2SS hereafter) is compatible with existing X-ray data but that the stars in CFL phase (CSS hereafter) cool down too rapidly, which disagrees with the data. However, in those calculations an important factor, as described below, is ignored.

An SS, both in normal phase and in color superconducting phase, can sustain a tiny nuclear crust with a maximum density below neutron drip $\left(\sim 10^{11} \mathrm{~g} \mathrm{~cm}^{-3}\right)$ and mass $M_{\mathrm{c}} \leq 10^{-5} M_{\odot}$ due to the existence of a strong electric field on the quark surface (Alcock et al. 1986; Usov 2004; Zheng \& Yu 2006). The spin-down of the star makes the matter at the bottom of the crust compress. As soon as the density exceeds neutron drip, the surplus matter in the crust falls into the quark core in the form of neutrons. Consequently, the engulfed neutrons dissolve into quarks, and the released energy during this process leads to 
a so-called deconfinement heating (DH). Yuan \& Zhang (1999) claim that DH delays the cooling of SSs in normal phase (NSS) and may even lead to a slight increase in the temperature at the early ages of a star under specific conditions.

We argue that the heating effect on the thermal evolution of stars in color superconducting phase is huge compared to NSSs because of the suppression of specific heat and neutrino emission involving pairing quarks. Therefore, focus on the effects of DH on the cooling of 2SSs and CSSs in this paper. Our paper is arranged as follows. We recall neutrino emissivities and specific heat, color superconductivity, and the DH mechanism in Sects. 2-4, respectively. The cooling curves and the corresponding explanations are presented in Sect. 5. Section 6 contains our conclusion and discussions.

\section{Neutrino emissivities and specific heat}

The emissivity associated with the QDU processes $d \rightarrow u e \bar{v}$ and $u e \rightarrow d v$ of quarks is (Iwamoto 1982)

$\epsilon^{(\mathrm{D})} \simeq 8.8 \times 10^{26} \alpha_{\mathrm{c}}\left(\frac{\rho_{\mathrm{b}}}{\rho_{0}}\right) Y_{\mathrm{e}}^{1 / 3} T_{9}^{6} \mathrm{erg} \mathrm{cm}^{-3} \mathrm{~s}^{-1}$,

where $\alpha_{\mathrm{c}}$ is the strong coupling constant, $\rho_{\mathrm{b}}$ is baryon density and $\rho_{0}=0.17 \mathrm{fm}^{-3}$ the nuclear saturation density, and $T_{9}$ is the temperature in units of $10^{9} \mathrm{~K}$. The electron fraction $Y_{\mathrm{e}}=\rho_{\mathrm{e}} / \rho_{\mathrm{b}}$ is small and even vanishes at a certain set of parameters of $\rho_{\mathrm{b}}, \alpha_{\mathrm{c}}$ and s-quark mass $m_{\mathrm{s}}$ (Duncan et al. 1983). It has not yet been considered that the contribution to the emissivity by the $s-u$ reaction, which is suppressed by an extra factor $\sin ^{2} \theta_{\mathrm{c}} \sim 10^{-3}$ compared to the $d-u$ reaction (Duncan et al. $1983)$, where $\theta_{\mathrm{c}}$ is the Cabibbo angle. When the QDU processes are switched off due to a small electron fraction $\left(Y_{\mathrm{e}}<Y_{\mathrm{ec}}=\right.$ $(3 / \pi)^{1 / 2} m_{\mathrm{e}}^{3} \alpha_{\mathrm{c}}^{-3 / 2} / 64$.), the contribution to the emissivities by the QMU processes $d q \rightarrow u q e \bar{v}$ and QB processes $q_{1} q_{2} \rightarrow q_{1} q_{2} v \bar{v}$ dominate. These emissivities were also estimated as (Iwamoto 1982)

$\epsilon^{(\mathrm{M})} \simeq 2.83 \times 10^{19} \alpha_{\mathrm{c}}^{2}\left(\frac{\rho_{\mathrm{b}}}{\rho_{0}}\right) T_{9}^{8} \operatorname{erg~cm}^{-3} \mathrm{~s}^{-1}$,

$\epsilon^{(\mathrm{B})} \simeq 2.98 \times 10^{19}\left(\frac{\rho_{\mathrm{b}}}{\rho_{0}}\right) T_{9}^{8} \mathrm{erg} \mathrm{cm}^{-3} \mathrm{~s}^{-1}$.

In order to compute the thermal evolution of the stars, we also need the the specific heat involving quarks and electrons written as (Iwamoto 1982; Blaschke et al. 2000)

$$
\begin{aligned}
c_{\mathrm{q}} & \simeq 2.5 \times 10^{20}\left(\frac{\rho_{\mathrm{b}}}{\rho_{0}}\right)^{2 / 3} T_{9} \mathrm{erg} \mathrm{cm}^{-3} \mathrm{~K}^{-1}, \\
c_{\mathrm{e}} & \simeq 0.6 \times 10^{20}\left(\frac{Y_{\mathrm{e}} \rho_{\mathrm{b}}}{\rho_{0}}\right)^{2 / 3} T_{9} \mathrm{erg} \mathrm{cm}^{-3} \mathrm{~K}^{-1} .
\end{aligned}
$$

Since the mass of the crust is very small, $M_{\mathrm{c}} \leq 10^{-5} M_{\odot}$, compared with the total mass of the star, its contribution to neutrino emissivity and specific heat can be neglected (Gudmundsson et al. 1983; Lattimer et al. 1994). Here we also ignore the neutrino emissivity and specific heat due to the photon-gluon excitation, because this excitation is only important for a temperature higher than $70 \mathrm{MeV}$ (Blaschke et al. 2000), which is much higher than the typical temperature in our calculation.

\section{Color superconductivity}

It is widely accepted that the color superconducting phase is the real ground state of quantized chromodynamics at asymptotically large densities. At a certain range of the quark chemical potential the quark-quark interaction is attractive, driving the pairing between the quarks (Alford et al. 1998, 1999; Alford \& Reddy 2003; Rapp et al. 1998; Shovkovy 2004). Because of the pairing, QDU processes are suppressed by a factor $\exp \left(-\Delta / k_{\mathrm{B}} T\right)$, and QMU \& QB processes are suppressed by a factor $\exp \left(-2 \Delta / k_{\mathrm{B}} T\right)$ for $T<T_{\mathrm{c}} \simeq 0.4 \Delta / k_{\mathrm{B}}$ (Blaschke et al. 2000). In the 2SC phase, two color states of $u$ and $d$ quarks pair, whereas the $s$ quark is unpaired. To be specific, we suppose that blue-green and green-blue $u-d$ quarks are paired, whereas red $u$ and $d$ quarks $\left(u_{\mathrm{r}}, d_{\mathrm{r}}\right)$ remain unpaired. As a consequence, the QDU processes on the red (unpaired) quarks, as $d_{\mathrm{r}} \rightarrow u_{\mathrm{r}} e \bar{v}$, as well as QMU, $d_{\mathrm{r}} q_{\mathrm{r}} \rightarrow u_{\mathrm{r}} q_{\mathrm{r}} e \bar{v}$, and QB, $q_{1 \mathrm{r}} q_{2 \mathrm{r}} \rightarrow q_{1 \mathrm{r}} q_{2 \mathrm{r}} \bar{v} \nu$, are not blocked, whereas other processes involving paired quarks are blocked out by a large pairing gap. Therefore, it can be estimated that the neutrino emissivities in 2SC phase are reduced by about one magnitude (Blaschke et al. 2000). On the other hand, the neutrino processes involving all flavors are suppressed in the CFL phase by the exponential factors. For both phases where the specific heat contributed by the paired quarks is also changed, we apply the formula (Blaschke et al. 2000)

$$
\begin{aligned}
c_{s \mathrm{q}}= & 3.2 c_{\mathrm{q}}\left(\frac{T_{\mathrm{c}}}{T}\right) \\
& \times\left[2.5-1.7\left(\frac{T}{T_{\mathrm{c}}}\right)+3.6\left(\frac{T}{T_{\mathrm{c}}}\right)^{2}\right] \exp \left(-\frac{\Delta}{k_{\mathrm{B}} T}\right) .
\end{aligned}
$$

\section{Deconfinement heating}

The effect of DH is determined by the number of neutrons engulfed by the quark core, in other words, the variation in the mass of the crust. The total heat released per time unit as a function of $t$ is

$H_{\mathrm{dec}}(t)=-q_{\mathrm{n}} \frac{1}{m_{\mathrm{b}}} \frac{\mathrm{d} M_{\mathrm{c}}}{\mathrm{d} v} \dot{v}$,

where $q_{\mathrm{n}}$, the heat release per absorbed neutron, is expected to be in the range $q_{\mathrm{n}} \sim 10-30 \mathrm{MeV}$ (Haensel \& Zdunik 1991), and $m_{\mathrm{b}}$ is the mass of baryon. Assuming the spin-down is induced by the magnetic dipole radiation, the evolution of the rotation frequency $v$ is given by

$\dot{v}=-\frac{8 \pi^{2}}{3 I c^{3}} \mu^{2} v^{3} \sin ^{2} \theta$,

where $I$ is the stellar moment of inertia, $\mu=\frac{1}{2} B R^{3}$ is the magnetic dipole moment, and $\theta$ is the inclination angle between magnetic and rotational axes. The mass of the crust $M_{\mathrm{c}}$ is calculated by a quadratic function of $v$ by Glendenning $\&$ Weber (1992), whose result describes the cases with intermediate frequencies $(\leq 500 \mathrm{~Hz})$ very well. Zdunik et al. (2001) improve the calculation nearly up to the Keplerian frequency using a polynomial including terms of higher order in $v$. The mass of the crust reads (Zdunik et al. 2001)

$M_{\mathrm{c}}=M_{\mathrm{c}}^{0}\left(1+0.24 v_{3}^{2}+0.16 v_{3}^{8}\right)$, 
where $v_{3}=v / 10^{3} \mathrm{~Hz}$, and $M_{\mathrm{c}}^{0} \approx 10^{-5} M_{\odot}$ is the mass of the crust in the static case.

\section{Cooling curves}

The thermal evolution with DH of a star is determined by the equation

$C \frac{\mathrm{d} T}{\mathrm{~d} t}=-L_{v}-L_{\gamma}+H_{\mathrm{dec}}$

where $C$ is the total specific heat, $L_{v}$ the neutrino luminosity, and $L_{\gamma}$ the surface photon luminosity given by

$L_{\gamma}=4 \pi R^{2} \sigma T_{\mathrm{s}}^{4}$,

where $\sigma$ is the Stefan-Boltzmann constant and $T_{\mathrm{S}}$ the surface temperature. The internal structure of SSs can be regarded as temperature-independent (Glen \& Sutherland 1980), and the surface temperature is related to internal temperature by a coefficient determined by the scattering processes occurring in the crust. In the work of Blaschke et al. (2000), this relation is given by a simple expression $T_{\mathrm{s}}=5 \times 10^{-2} T$, but following Yuan \& Zhang (1999), we apply an accurate formula that is demonstrated by Gudmundsson et al. (1983),

$T_{\mathrm{s}}=3.08 \times 10^{6} g_{\mathrm{s}, 14}^{1 / 4} T_{9}^{0.5495}$,

where $g_{\mathrm{s}, 14}$ is the proper surface gravity of the star in units of $10^{14} \mathrm{~cm} \mathrm{~s}^{-2}$; or for a recent version see the result of Potekhin et al. (1997).

In our calculations, to be specific, we consider a model of canonical SS of $1.4 M_{\odot}$ at a constant density, which is a very good approximation for SSs of mass $M \leq 1.4 M_{\odot}$ (Alcock et al. 1986). As used by Blaschke et al. (2000), we take $Y_{\mathrm{e}}=10^{-5}$, $\alpha_{\mathrm{c}}=0.25, \rho=3 \rho_{0}$ for $Y_{\mathrm{e}}>Y_{\mathrm{ec}}$, which is a representative set of parameters for which the QDU processes contribute to the cooling, whereas $Y_{\mathrm{e}}=0, \alpha_{\mathrm{c}}=0.15, \rho=5 \rho_{0}$ for $Y_{\mathrm{e}}<Y_{\mathrm{ec}}$. And we also choose $\Delta=100 \mathrm{MeV}$ and $q_{\mathrm{n}}=20 \mathrm{MeV}$, the initial temperature $T_{0}=10^{9} \mathrm{~K}$, initial period $P_{0}=0.78 \mathrm{~ms}$, and the magnetic tilt angle $\theta=45^{\circ}$. The gravitational red-shift is also taken into account. Then the effective surface temperature detected by a distant observer is $T_{\mathrm{s}}^{\infty}=T_{\mathrm{s}} \sqrt{1-R_{\mathrm{g}} / R}$, where $R_{\mathrm{g}}$ is the gravitational stellar radius.

We plot the cooling curves without DH of NSSs (solid curves), 2SSs (dotted curves), and CSSs (dashed curves) in Fig. 1. And the observational data, which are taken from Tables 1 and 2 in Page et al. (2004), are also shown in order to give the readers a feeling of the position of the illustrative curves in the logarithm $T_{\mathrm{s}}^{\infty}-t$ plane. But we will not try to fit the data carefully in this paper. It shows that the cooling history of 2SSs are similar to NSSs, whereas CSSs become very cold at an early age since the specific heat is very small, cooler than $10^{4.5} \mathrm{~K}$ after $1000 \mathrm{yr}$ for $Y_{\mathrm{e}}=10^{-5}$ or several hours for $Y_{\mathrm{e}}=0$ (this curve is not shown in Fig. 1). We can see the curves of CSSs are very far from the data. These conclusions are also indicated by Blaschke et al. (2000) using the relation of $T_{\mathrm{s}}=5 \times 10^{-2} \mathrm{~T}$.

Figure 2 shows the cooling behaviors of 2SSs with DH for various magnetic fields $\left(10^{8}-10^{12} \mathrm{G}\right)$. And the analogs

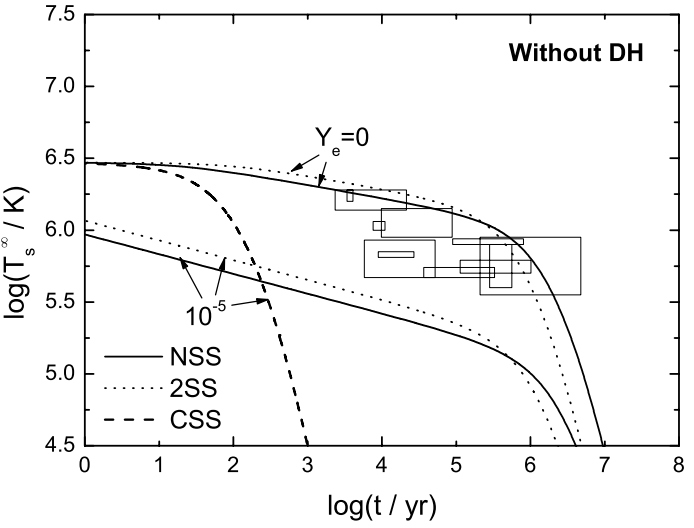

Fig. 1. Cooling curves of NSSs, 2SSs, and CSSs without DH.

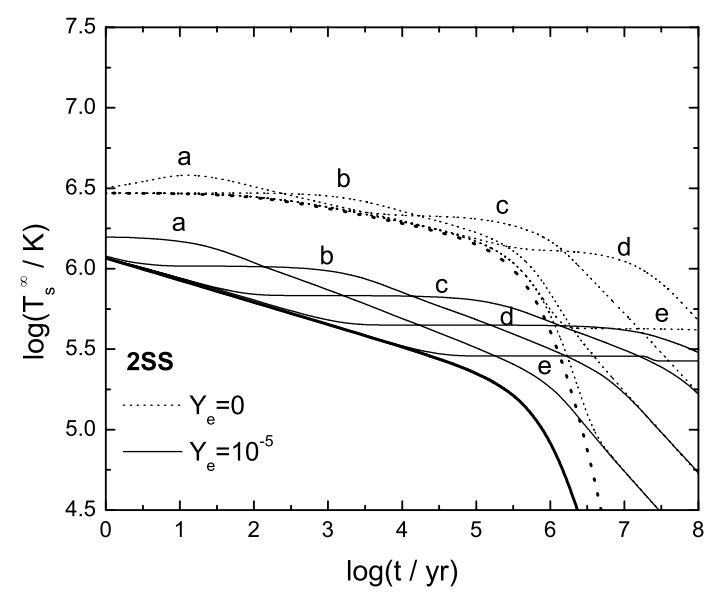

Fig. 2. Cooling curves of 2SSs with $\mathrm{DH}$ for various magnetic fields (curve a: $10^{12} \mathrm{G}, \mathrm{b}: 10^{11} \mathrm{G}, \mathrm{c}: 10^{10} \mathrm{G}, \mathrm{d}: 10^{9} \mathrm{G}$, e: $10^{8} \mathrm{G}$ ) and the curves without DH (thick curve).

for NSSs can be seen in Yuan \& Zhang (1999). We can see DH delays the stellar cooling considerably. As discussed by Yuan \& Zhang (1999), the stronger the magnetic field the more rapid the spin-down, and most of the nuclear matter in the crust dissolves during an earlier and shorter time. For a 2SS with a strong $\left(B=10^{12} \mathrm{G}\right)$ field and small electron fraction (dotted curve a), a distinct heating period exists in the first several ten years. And in the cases of weak fields $\left(B<10^{10} \mathrm{G}\right)$, stars could maintain high temperatures even at older ages $\left(>10^{6} \mathrm{yr}\right.$ ). In the following paragraph, we discuss in detail how $\mathrm{DH}$ induces temperature rise and delays cooling.

We here pay more attention to the situation of CSSs because we argue the existence of a marked heating effect relative to the reduced emissions. To be clear, Fig. 3 shows the cooling curves of CSSs with DH for both a strong $\left(B=10^{11} \mathrm{G}\right)$ and a weak $\left(B=10^{9} \mathrm{G}\right)$ magnetic field. It is obvious that the cooling curves are changed dramatically by DH. The strong magnetic field induces a rapid spin-down of the star at the earliest ages, which could enhance the effect of DH to make it greater than the cooling effect at the beginning. As a result, the temperature should rise due to the surplus heat until the increasing luminosity equals the heating effect, $L_{\gamma}=H_{\mathrm{dec}}$, so a net heating period appears at the earliest ages. On the other hand, the temperature of the star with a weak field decreases 


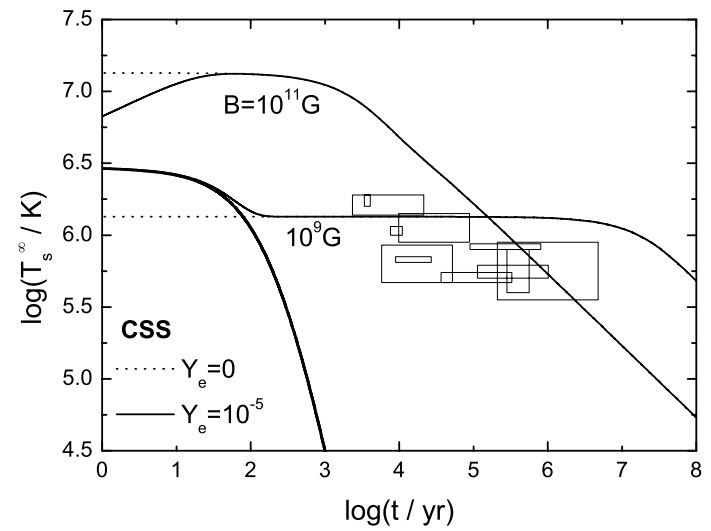

Fig. 3. Cooling curves of CSSs with DH for a strong $\left(B=10^{11} \mathrm{G}\right)$ and a weak $\left(B=10^{9} \mathrm{G}\right)$ magnetic field and the curve without DH (thick curve).

but does not rise, due to the relative greater cooling effect at the start until the thermal release is compensated for by DH entirely: $L_{\gamma}=H_{\mathrm{dec}}$. In this case, since the confinement energy deposited in the crust is released slowly to heat the star, the star with a weak field can maintain a high temperature even at older ages $\left(>10^{6} \mathrm{yr}\right)$. To conclude, both the stars with strong and weak fields, after several hundred years (the specific value of the time is determined by the specific condition of the star), could arrive at an equilibrium between the cooling and heating effects. From then on, the temperature could only be reduced in order to rebuild the equilibrium when $H_{\mathrm{dec}}$ deceases with time, so the cooling of the stars is delayed. Due to this delay, the curves of CSSs cannot be in conflict with observational data as shown in Fig. 3. Since the neutrino emission involving all quarks in CSSs is suppressed, the equilibrium discussed above is only determined by DH and photon emission, and has hardly anything to do with the interior thermal properties of the star. Therefore, the dependence of the cooling on the electron fraction is eliminated after the first several hundred years. Going back to Fig. 2, we see that the mechanism described above also influences the cooling of 2SSs. However, since the equilibrium $\left(L_{v}+L_{\gamma}=H_{\mathrm{dec}}\right)$ should involve neutrino luminosity, which is larger at high temperature whereas smaller at low temperature than photon luminosity, the evolution of 2SSs may be more complicated than it is for CSSs (see Fig. 4 for detail), i.e., the cooling history can be roughly divided into a neutrino cooling stage $\left(L_{v} \gg L_{\gamma}\right)$ and a photon cooling stage $\left(L_{v} \ll L_{\gamma}\right)$, and the cooling is sensitive to the electron fraction; and the moment when the equilibrium is achieved could be very different for different magnetic fields.

Figure 4 shows the cooling curves of CSSs with different magnetic fields. We can see the cooling of the stars with any field is delayed, just as we find in Fig. 2. However, in comparison with Fig. 2, there is a question of why the temperature rise of CSSs can be more significant than the one of 2SSs. For both CSSs and 2SSs, as discussed in the previous paragraph, the reason for the temperature rise is that the heating effect is greater than the cooling effect at the beginning: $H_{\mathrm{dec}}>L_{\gamma}$ for CSSs and $H_{\mathrm{dec}}>L_{v}+L_{\gamma}$ for 2SSs. Since the neutrino term is absent for CSSs, the initial difference between the heating term and

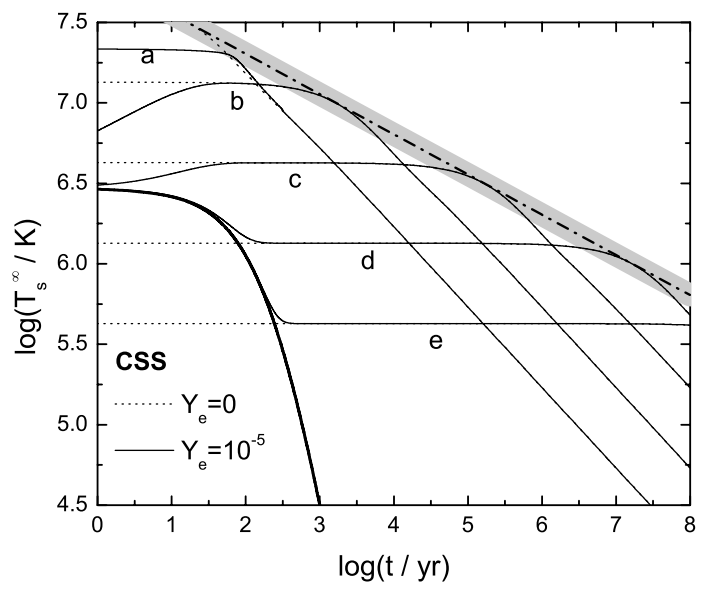

Fig. 4. The same as Fig. 2 but for CSSs. The dash-dotted curve is the limit line, and the band-like structure is supposed to reflect the uncertainty of $q_{\mathrm{n}}$.

luminosity of CSSs is much larger than the one of 2SSs with the same magnetic field and initial temperature. On the other hand, with the rise in temperature, the increase in the luminosity is proportional to $T^{2.2}$ for CSSs (see Eqs. (11), (12)) but to $T^{8}$ for 2SSs with a small electron fraction (see Eqs. (2), (3), where the term of photon luminosity is ignored since $L_{v} \gg L_{\gamma}$ at high temperature). Therefore, 2 SSs can achieve the equilibrium easily after a comparatively small temperature rise, but the magnitude of the rise for CSSs needs to be much larger. In addition, the needed magnetic field intensity to induce the temperature rise of CSSs could be smaller than 2SSs since the initial cooling effect of CSSs is smaller.

We link the points where the cooling curves turn down together as a line (dash-dotted curve) in logarithm $T_{\mathrm{s}}^{\infty}-t$ plane in Fig. 4. The temperature indicated by the line is expressed as a power form $T_{\mathrm{s}, \mathrm{lim}}^{\infty}=6.4 \times 10^{7} t^{-1 / 4} \mathrm{~K}$. It may be well-founded that any other cooling curves of isolated stars (to our knowledge), regardless of star models, will be below this line due to the high heating effect and low cooling effect in CSSs. Hence we reckon that the indicated temperature may be the upper limit to what compact stars can reach at a given age. We must emphasize that this line found from the cooling curves of CSSs is only determined by the equilibrium between $\mathrm{DH}$ and surface photon cooling.

Finally, we present the cooling curves of CSSs for different gaps in Fig. 5. It can be seen that the cooling curves are almost independent of the gap on a very large parameter scale $(\Delta \sim 10-100 \mathrm{MeV})$.

\section{Conclusion and discussions}

We have studied the cooling behaviors of rotating SSs in the presence of color superconductivity by considering the effect of DH. The thermal evolution of SSs is now quite different from previous results, because DH can delay the cooling, and color superconductivity enhances this effect significantly, especially in the CFL phase. For CSSs, the previous discussions point out that the specific heat is determined by electrons since the contribution of quarks has been suppressed. This reduction leads 


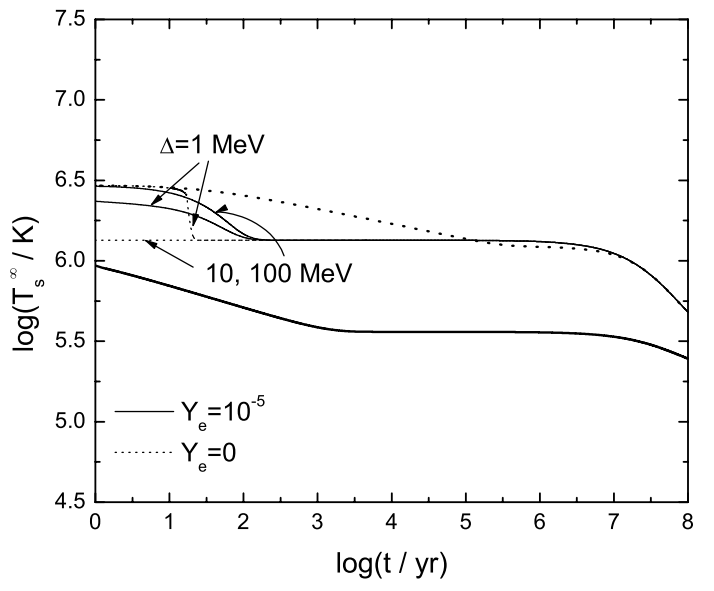

Fig. 5. Cooling curves of CSSs with $B=10^{9} \mathrm{G}$ for different gaps. The thick curves correspond to NSSs.

to a very rapid cooling that disagrees with observational data (Blaschke et al. 2000). However, when we consider the effect of $\mathrm{DH}$, the results should imply that the cooling curves could not be in serious conflict with the data. We even find it is possible that CSSs reach a higher temperature than other kinds of compact stars in their cooling history. The limit temperature line should illustrate this conjecture.

To be specific, as pointed out by Yuan \& Zhang (1999), a temperature rising period could exist at the early ages due to the DH with a strong magnetic field. And we argue that the presence of color superconductivity may lead to a significant rise. Yuan \& Zhang (1999) propose that this phenomena may be a signature of the existence of SS. In our opinion, if the theory of the color superconductivity is reliable, observing a young and quite hot source may be possible, although we also note that an important so-called brightness constraint has been suggested recently by Grigorian (2005), who argues that it is unlikely that objects with a given age are hotter than those already observed. Statistically, this constraint is a good finding, but we think that in theory the possibility of the existence of young hotter stars still cannot be rejected absolutely. Of course, for our model, the early evolution also may be changed to a certain extent if we consider the formation of the crust of the star. On the other hand, for those stars with weak fields $\left(<10^{10} \mathrm{G}\right)$, our results show that they can maintain a high temperature at older ages $\left(>10^{6} \mathrm{yr}\right)$. Unfortunately, these older sources with weak fields also have been not detected up to now (see, for example, Popov et al. (2003) for the list of close-by cooling pulsars). To summarize, at the present point of observations, there is no evidence of the existence of extra hot sources. It may imply that the model needs some further improvements. However, it still should be emphasized that the various heating mechanisms in compact stars need to be given more importance when we talk about the star's cooling when using the so-called standard scenario.

Acknowledgements. We would like to thank Prof. D. F. Hou for the useful discussion. We are especially indebted to the anonymous referee for his/her useful comments that helped us to improve the paper. This work was supported by the NFSC under Grant Nos. 10373007 and 90303007.

\section{References}

Alcock, C., Farhi, E., \& Olinto, A. 1986, ApJ, 310, 261

Alcock, C., \& Olinto, A. 1988, Ann. Rev. Nucl. Sci., 38, 161

Alford, M. 2004, J. Phys. G, 30, 441

Alford, M., Rajagopal, K., \& Wilczek, F. 1998, Phys. Lett. B, 422, 247

Alford, M., Rajagopal, K., \& Wilczek, F. 1999, Nucl. Phys. B, 537, 443

Alford, M., \& Reddy, S. 2003, Phys. Rev. D, 67, 074024

Blaschke, D., \& Klähn, T., \& Voskresensky, D. N. 2000, ApJ, 533, 406

Duncan, R. C., Shapiro, S. L., \& Wasserman, I. 1983, ApJ, 267, 358

Glen, G., \& Sutherland, P. 1980, ApJ, 239, 671

Glendenning, N. K., \& Weber, F. 1992, ApJ, 400, 647

Grigorian, H., Blaschke, D., \& Voskresensky, D. 2005, Phys. Rev. C, 71,045801

Grigorian, H. 2005 [arXiv: astro-ph/0507052]

Gudmundsson, E. H., Pethick, C. J., \& Epstein, R. I. 1983, ApJ, 272, 286

Haensel, P., \& Zdunik, J. 1991, in Strange Quark Matter in Physics and Astrophysics, ed. J. Madsen, \& P. Haensel (Nucl. Phys. B [Proc. Suppl.], 24), 139

Iwamoto, N. 1982, Ann. Phys., 141, 1

Lattimer, J. M., Van Riper, K. A., Prakash, M., \& Prakash, M. 1994, ApJ, 425, 802

Page, D. 1992, in Proc. of the work-shop on High Energy Phenomenology, ed. M. Péréz, \& R. Huerta (Singapore: World Scientific), 347

Page, D., Lattimer, J. M., Prakash, M., \& Steiner, A. W. 2004, ApJS, 155,623

Pizzochero, P. M. 1991, Phys. Rev. Lett., 66, 2425

Potekhin, A. Y., Chabrier, G., \& Yakovlev, D. G. 1997, A\&A, 323, 415

Popov, S. B., Colpi, M., Prokhorov, M. E., Treves, A., \& Turolla, R. 2003, A\&A, 406, 111

Rapp, R., Schäfer, T., Shuryak, E. V., \& Velkovsky, M. 1998, Phys. Rev. Lett., 81, 53

Schaab, C., Hermann, B., Weber, F., \& Weigel, M. K. 1997a, ApJ, 480, L111

Schaab, C., Hermann, B., Weber, F., \& Weigel, M. K. 1997b, J. Phys. G, 23, 2029

Schaab, C., Weber, F., Weigel, M. K., \& Glendenning, N. K. 1996, Nucl. Phys. A, 605, 531

Shovkovy, I. A. 2004, lectures delivered at the IARD 2004 conference, Saas Fee, Switzerland, June 12-19, and at the Helmholtz International Summer School and Workshop on Hot points in Astrophysics and Cosmology, JINR, Dubna, Russia, Aug. 2-13 [arXiv:nucl-th/0410091]

Shovkovy, I. A., \& Ellis, P. J. 2002, talk presented at workshop, Continuous Advances in QCD 2002/Arkadyfest, Minneapolis, USA, May, 17-23 [arXiv: astro-ph/0207346]

Usov, V.V. 2004, Phys. Rev. D, 70, 067301

Yuan, Y. F., \& Zhang, J. L. 1999, A\&A, 344, 371

Zdunik, J. L., Haensel, P., \& Gourgoulhon, E. 2001, A\&A, 372, 535

Zheng, X. P., \& Yu, Y. W. 2006, A\&A, 445, 627 